\title{
Traitement de trois images M.E.B. issues de la même scène : une aide à l'expertise de monuments historiques
}

\author{
Rachid Harba $\left({ }^{1}\right)$, Gérard Jacquet $\left({ }^{1}\right)$ et Michel Rautureau $\left({ }^{2}\right)$ \\ (1) Groupe de Recherche sur l'Energétique des Milieux Ionisés et G.D.R. 134 Traitement du signal \\ et des images, U.F.R. Faculté des Sciences, rue de Chartres BP 6759, 45067 Orléans Cedex 2, \\ France \\ $\left(^{2}\right)$ Centre de Recherche sur la Matière Divisée, Laboratoire de Cristallographie, U.F.R. Faculté des \\ Sciences, rue de Chartres BP 6759, 45067 Orléans Cedex 2, France
}

(Reçu le 23 juillet 1991, accepté le 28 octobre 1991)

\begin{abstract}
Résumé. - Cet article décrit une méthode de traitement de trois images, issues de la même scène, obtenues à partir d'un microscope électronique à balayage (M.E.B.). Ces trois images sont complémentaires comme l'est un puzzle. L'échantillon analysé est une coupe pétrographique d'une pierre utilisée pour la construction de monuments historiques. Le but de l'étude est d'extraire deux classes de particules (calcaire et siliceuse) et de visualiser la porosité de la pierre afin de réaliser une aide à l'expertise. Une stratégie adaptée à la qualité des images est mise en place. Un premier seuil sur l'image de bonne qualité est déterminé et permet de séparer la porosité des constituants. Les deux autres seuils sont obtenus en utilisant la complémentarité des trois images. La segmentation ainsi obtenue permet de calculer le pourcentage des composants et d'analyser l'aspect géométrique des particules ; l'interprétation de ces premiers résultats permet de mieux apprécier l'état de la pierre.
\end{abstract}

\begin{abstract}
This paper describes a three complementary images processing method. These three scanning electron microscope images are taken from a petrographic slice of stone used to build historical monuments. The goal is to separate two classes of particles (calcareous and siliceous) in the stone to improve image interpretation. A specific process is developed: a first threshold on the good quality image allows the separation between particles and porosity. The complementary of the three images gives the other thresholds. Primary results on components percentages and geometrical shape are presented. They allow a better knowledge of the analysed stone.
\end{abstract}

\section{Introduction.}

Lorsqu'un monument historique se dégrade au cours du temps ou sous l'action d'agents extérieurs, un expert étudie l'état du bâtiment. Pour ce faire, la proportion des différents composants des pierres de la construction ainsi que le pourcentage et la nature de la porosité sont des paramètres fondamentaux. Ces renseignements sont obtenus à différentes échelles de précision par pesage, par une analyse chimique ou par une analyse élémentaire (énergie des Rayons X émis) faite en microscopie électronique à balayage (M.E.B.) [1]. En comparant les résultats à des valeurs 
antérieures obtenues sur le même édifice ou à des caractéristiques de base des pierres (pierres de carrière) l'expert aura des renseignements sur l'état du bâtiment. De plus, les éventuelles pierres de remplacement doivent être de même nature que celles d'origine car il ne faut pas perturber la circulation de l'eau à l'intérieur du matériau. Il est donc utile de connaître l'anisotropie et la proportion des zones poreuses. Ces parametres permettent de chiffrer le sens et la facilité de l'écoulement de l'eau et d'approcher les caractéristiques de perméabilité et de tortuosité de la pierre. D'autre part, une étude granulométrique de ses composants donne des informations complémentaires sur sa solidité. Une analyse par traitement numériques d'images permet de chiffrer ces paramètres.

La présente étude traite des premiers résultats obtenus sur le "Tuffeau" qui est la pierre la plus employée dans les édifices du Val de Loire (France). Cette pierre détritique, très poreuse, est essentiellement composée de particules calcaires et siliceuses. La procédure pour les analyser par M.E.B. est décrite au chapitre 2. Ainsi, trois films complémentaires mettant chacun en évidence une composante de la pierre vont permettre d'extraire les différentes particules et la porosité par seuillage des images. Le chapitre 3 décrit plusieurs méthodes de segmentation appliquées à l'image porosité qui, étant de meilleure qualité, va être utilisée pour séparer, dans un premier temps, les particules de la porosité. La séparation des particules entre elles, basée sur l'hypothèse de complémentarité, fait l'objet du chapitre 4. Les résultats obtenus sont présentés chapitre 5 ; les informations issues de l'exploitation des images segmentées sont décrites dans la dernière partie où nous montrons comment les relier à des caractéristiques utiles pour un expert.

\section{Images en microscopie électronique.}

Les échantillons étudiés en M.E.B. sont des lames pétrographiques, dont la surface est polie, de quelques centimètres carrés et d'épaisseur standard 30 micromètres. L'observation se fait perpendiculairement à la surface. La porosité ouverte est remplie de résine synthétique. Cette résine est choisie pour sa grande fluidité et sa capacité à être observée par fluorescence en microscopie optique. L'induration est nécessaire car le tuffeau est un matériau très polyphasé peu cohérent. Dans son ensemble, ce type d'échantillon est mauvais conducteur électrique et la résine d'inclusion isolante. L'épaisseur de trente micromètres est très grande devant la profondeur de pénétration qui est nettement inférieure à un micromètre [2,3]. Ces échantillons, analysés au microscope électronique à balayage, donnent un film où seuls les électrons rétrodiffusés ont été pris en compte. Cette image de bonne qualité est appelée image porosité (Fig. 1a) ; elle est composée de nombreuse particules sombres qui se détachent de la porosité très claire. On peut différencier ces particules :

- les zones noires correspondent à de la calcite, de petits fossiles calcaires et des particules de quartz ;

- les foncées se rapportent essentiellement au silicium (quartz ou mica);

- les plus claires sont formées de silice ou d'argile (phyllosilicates).

La répartition des niveaux de gris pour l'image porosité se traduit dans l'histogramme (Fig. 1b) par deux modes distincts : celui de niveaux de gris les plus faibles pour les particules et l'autre pour la porosité. Cette image ne permettra pas de séparer les particules calcaires et siliceuses car elles sont de niveaux de gris trop voisins. Sur tous les histogrammes présentés dans ce travail, on remarque un petit pic au niveau de gris 128 dû à une anomalie du convertisseur analogiquenumérique de la carte d'acquisition d'images. Cette anomalie est suffisamment faible pour ne pas perturber les méthodes de segmentation proposées. 


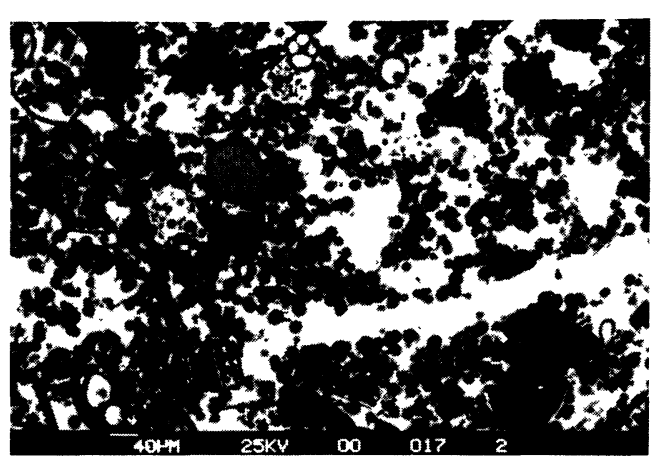

a)

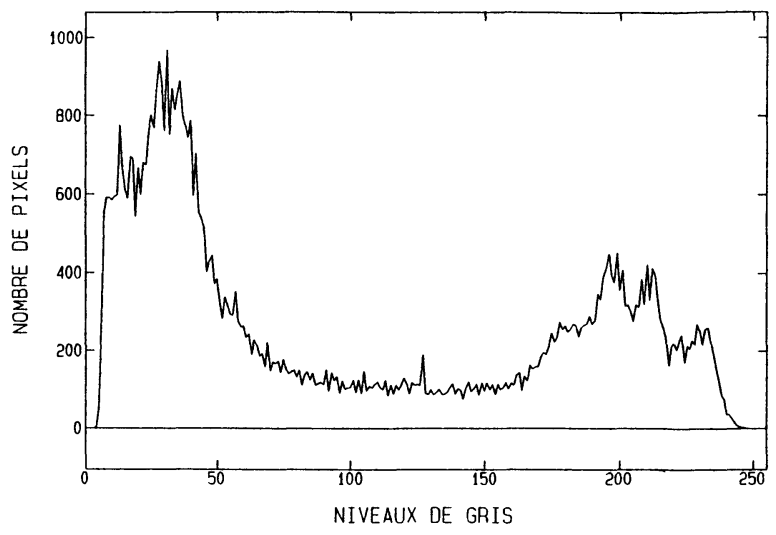

b)

Fig. 1. - a) Image montrant la porosité. b) Histogramme de l'image porosité.

[a) Porosity image. b) Histogram of the porosity image.]

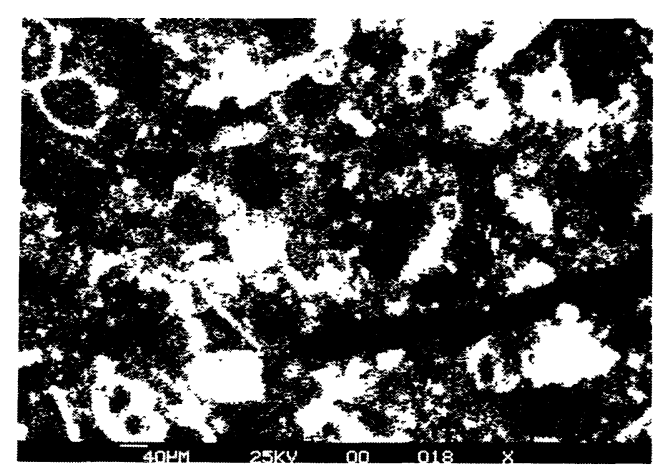

a)

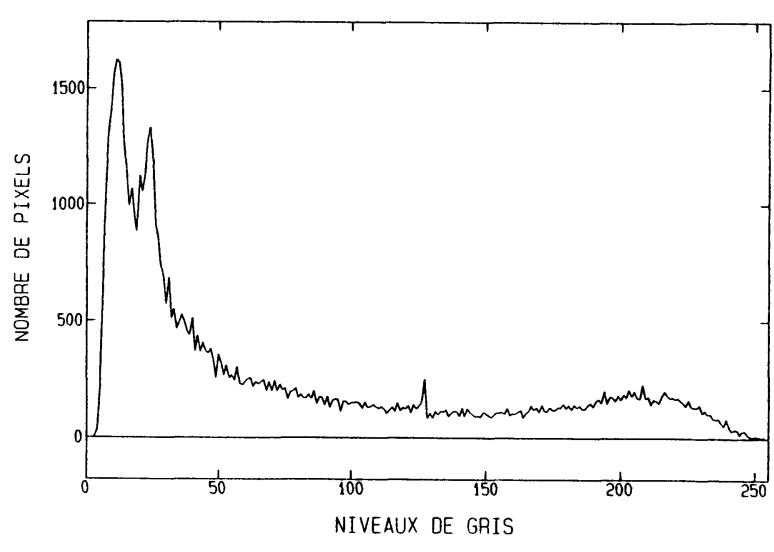

b)

Fig. 2. - a) Image calcium. b) Histogramme de l'image calcium.

[a) Calcium image. b) Histogram of the calcium image.]

En complément de cette analyse en électrons, le M.E.B. permet aussi d'étudier l'émission X de chaque élément. Deux images obtenues sur film polaroïd sont intéressantes :

- une image calcium (Fig. 2a) : les particules calcaires sont représentées par les zones claires de l'image, le reste est noir ;

- une image silicium (Fig. 3a) où les zones claires sont les particules siliceuses.

Le M.E.B. utilise ne fournit actuellement que des films polaroïd. Une zone de ces films est numérisée au format $256 \times 256$ pixels avec une caméra C.C.D.. Ce passage obligé provoque des pertes d'information. De même, il faut acquérir exactement la même zone sur les trois polaroïds. Le bon calage des photos est très important. Les histogrammes associés (Figs. $2 b$ et $3 b$ ) sont formés de deux modes : celui de droite correspond à l'élément analysé. La forme de ces trois histogrammes montre que des techniques de seuillage seront bien adaptées. 


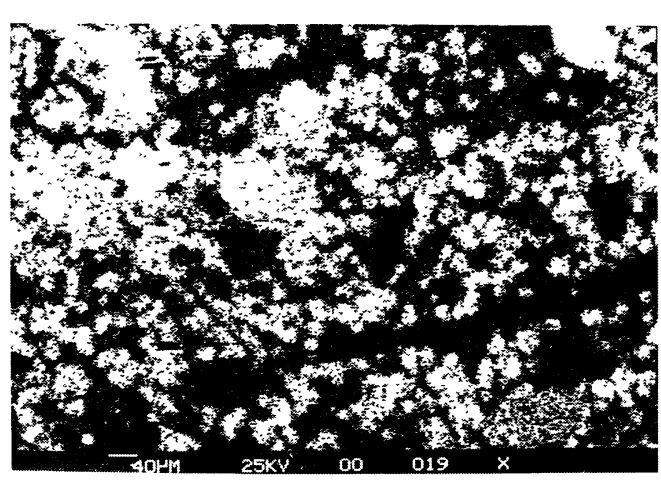

a)

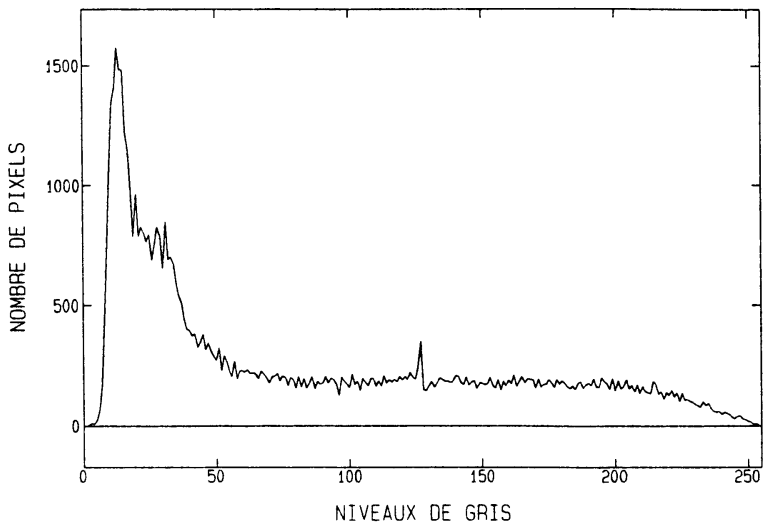

b)

Fig. 3. - a) Image silicium. b) Histogramme de l'image silicium.

[a) Silicon image. b) Histogram of the silicon image.]

D'un point de vue qualitatif, l'image en électrons rétrodiffusés est la meilleure. Le faible grandissement nécessaire pour l'observation d'une zone significative de l'objet (Figs. 1a, 2a et 3a) conduit à utiliser le microscope loin de sa résolution spatiale limite (quinze nanomètres dans le cas présent). De plus, la dimension de la sonde et le pas de balayage sont plus grands que cette limite. Les images $\mathrm{X}$ des zones calciques et siliciques ont une résolution moins bonne du fait de nombreux parametres : émission en volume suivie d'une absorption, effet d'orientation des particules dans la zone d'émission, etc. L'ensemble des phénomènes physiques ne peut être modélisé simplement à cause de la très grande variété des particules présentes dans l'échantillon. Sans ces perturbations, les trois images issues de la même scène (l'image électronique et les deux en Rayons X) devraient être complémentaires car les particules sont en quasi totalité soit calcaires soit siliceuses. Pour segmenter ces images, une stratégie adaptée à leur qualité va être présentée, elle consiste à séparer la porosité des particules puis à classer les particules en deux groupes : calcaires ou siliceuses.

\section{Séparation des particules de la porosité.}

Dans un premier temps, le seuil sur l'image porosité peut être déterminé avec une bonne précision par des méthodes usuelles. La segmentation de cette image permettra d'extraire les particules de la porosité. Parmi les méthodes de seuillage décrites par Sahoo et al. [4], trois ont été testées.

Dans ces trois méthodes, on estimera la probabilité d'apparition d'un pixel à un niveau de gris $i$ par:

$$
p_{i}=\frac{N_{i}}{N}
$$

avec $N_{i}$ le nombre de pixels au niveau $i$ et $N$ le nombre total de pixels.

3.1 MÉTHOdE DU MINIMUM D’ERREUR BAYÉSIENNE. - On suppose que la densité de probabilité d'apparition d'un pixel à un niveau de gris $i$ est la somme pondérée de deux distributions 
normales $p(i / j)$. On obtient alors :

$$
p_{i}=\sum_{j=1}^{2} W_{j} p(i / j)
$$

où les probabilités

$p(i / j)=\frac{1}{\sqrt{2 \pi} \sigma_{j}} \exp \left(-\frac{\left(i-\mu_{j}\right)^{2}}{2 \sigma_{j}^{2}}\right)$ sont pondérées par les poids $W_{j}$.

La méthode itérative proposée par Kittler et al. [5] permet de s'affranchir de l'estimation des paramètres $\sigma, \mu$ et de la pondération des deux modes. On choisit un seuil de départ qui sépare l'histogramme en deux distributions. On peut alors calculer moyennes, variances et poids respectifs. Un nouveau seuil $t$ est déterminé à chaque itération en minimisant l'erreur due au recouvrement partiel de ces distributions (règle du minimum d'erreur Bayésienne). Ce seuil est solution de l'equation :

$$
\frac{\left(t-\mu_{1}\right)^{2}}{\sigma_{1}^{2}}+\log _{\mathrm{e}} \sigma_{1}^{2}-2 \log _{\mathrm{e}} W_{1}=\frac{\left(t-\mu_{2}\right)^{2}}{\sigma_{2}^{2}}+\log _{\mathrm{e}} \sigma_{2}^{2}-2 \log _{\mathrm{e}} W_{2}
$$

Le seuil de convergence sera le seuil de binarisation.

3.2 MÉTHOde Du MAXIMUM D'ENTROPIE. - En théorie de l'information, on associe à un histogramme ou à la densité de probabilité associée une quantité $E$ que l'on appelle entropie $[6,7]$ :

$$
E=-\sum_{i=0}^{M-1} p_{i} \log _{2} p_{i}
$$

où $M$ est le nombre de niveaux de gris.

Plusieurs auteurs proposent des méthodes de segmentation qui maximisent l'entropie après seuillage. Ainsi, Kapur et al. [8] obtiennent deux distributions A et B (celle du fond et celle de l'objet) en séparant l'histogramme par un seuil $t$. On associe à ces distributions deux entropies :

$$
\begin{gathered}
E(\mathrm{~A})=-\sum_{i=0}^{t} \frac{p_{i}}{p_{t}} \log _{2}\left(\frac{p_{i}}{p_{t}}\right) \text { et } E(\mathrm{~B})=-\sum_{i=t+1}^{M-1} \frac{p_{i}}{1-p_{t}} \log _{2}\left(\frac{p_{i}}{1-p_{t}}\right) \\
\operatorname{avec} p_{t}=\sum_{i=0}^{t} p_{i} .
\end{gathered}
$$

Le maximum de $H$, somme de $E(\mathrm{~A})$ et de $E(\mathrm{~B})$, correspond au maximum d'information entre l'objet et le fond ; ce sera le seuil de binarisation. La forme de $H$ est illustrée figure 4. On peut voir que cette courbe est plate dans la zone d'intérêt, mais la détermination de la position du maximum reste précise ( \pm 2 niveaux de gris) car la courbe est très régulière.

3.3 Modification D'Histogramme. - L'idée de base décrite par Weszka et al. [9] est de dire que la meilleure séparation entre le fond et un objet dans une image est l'endroit où le gradient de niveaux de gris est le plus élevé. Pour mettre cette idée en oeuvre, on peut modifier l'histogramme en pondérant l'apport de chaque pixel par la valeur du gradient et faire apparaître un minimum qui sera le seuil de binarisation. Cette pondération a la valeur suivante :

$$
1 /\left(1+|\Delta|^{2}\right) \text { avec } \Delta \text { le gradient en un point considéré. }
$$

Les figures $5 \mathrm{a}, 5 \mathrm{~b}$ et $5 \mathrm{c}$ illustrent la méthode. Le seuil retenu est le minimum de l'histogramme modifié. Les variations locales étant importantes dans la zone utile (Fig. 5c), la position du minimum peut varier d'environ 10 niveaux de gris sur 256 d'une acquisition d'image à l'autre. 


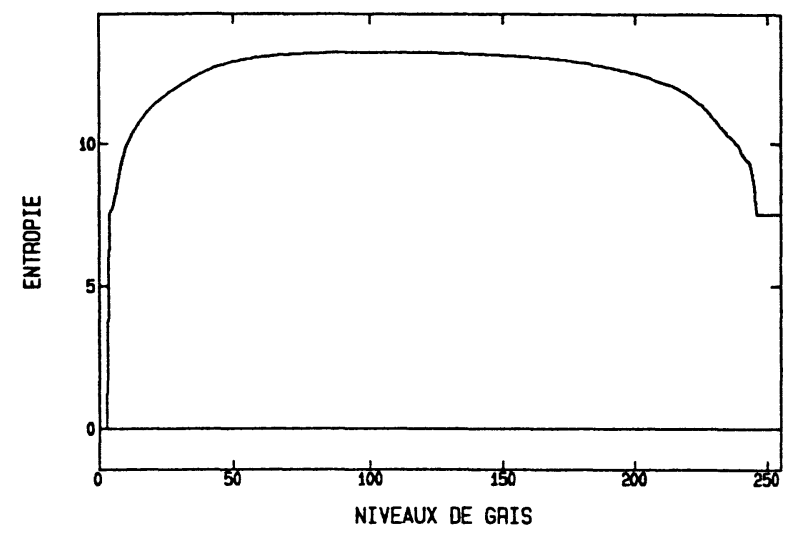

Fig. 4. - Valeur de l'entropie pour l'image porosité en fonction du niveau de gris de seuillage. [Value of the entropy of the porosity image vs. grey levels.]

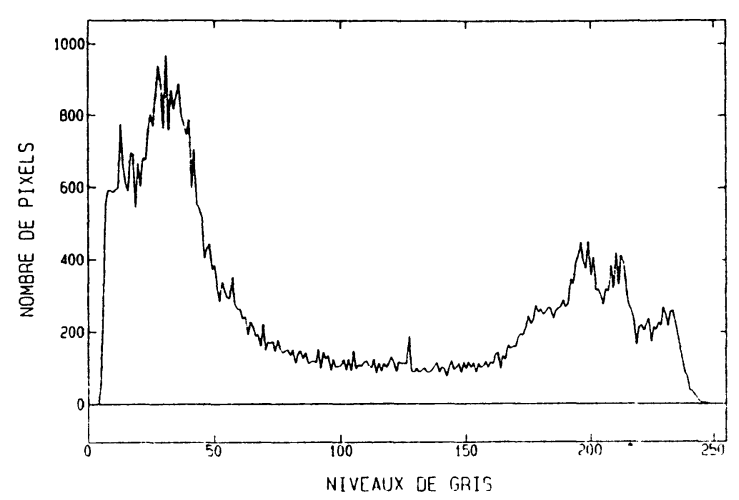

a)

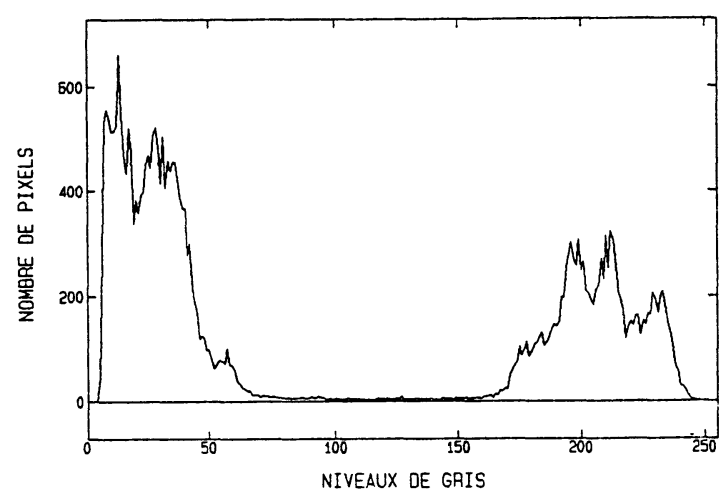

b)

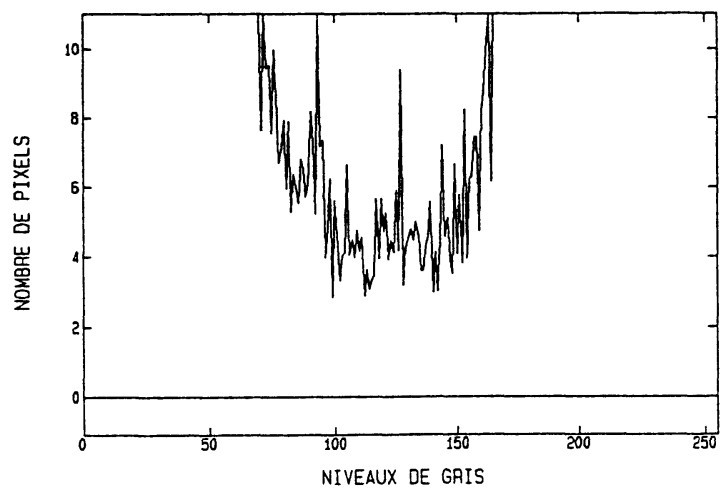

c)

Fig. 5. - a) Histogramme original de l'image porosité. b) Histogramme 5a modifié. c) Vue agrandie de la zone d'intérêt.

[a) Original histogram of the porosity image. b) Modified histogram. c) Enlargement of area of interest.] 
3.4 COMPARAISON DES MÉTHODES ET DISCUSSION. - Les trois méthodes ont été testées sur une série de 11 images issues d'une analyse en électrons (image porosité). Pour chacune des images, il a été demandé à une personne expérimentée de donner le seuil séparant au mieux les particules de la porosité. Nous avons pris ces seuils comme référence. Le tableau I donne l'écart moyen entre les seuils de référence et ceux trouvés par les trois méthodes sur les 11 images test.

Tableau I. - Ecart moyen pour les trois méthodes étudiées.

[Average error for the three different methods.]

\begin{tabular}{|l|c|c|c|}
\hline & Min. Err. & Max. En. & Mod.His. \\
\hline Ecart moy. & 24 & 21 & 41 \\
\hline
\end{tabular}

Dans notre cas, le maximum d'entropie conduit à l'erreur la plus faible entre les trois méthodes testées : celle-ci sera donc retenue par la suite.

\section{Séparation des particules en deux classes.}

Les trois images sont complémentaires. Cette information a priori va être utilisée afin de mettre en oeuvre une méthode de séparation des particules. En effet, en superposant les trois images après seuillage, on peut mettre en évidence un certain nombre de pixels mal classés qui ne respectent pas cette complémentarité. Ce nombre de pixels varie avec les valeurs des seuils ; son minimum correspondra à une segmentation optimale au sens du critère de superposition suivant : le seuil $T_{\mathrm{p}}$ de l'image porosité ayant déjà été déterminé afin de séparer la porosité des particules, nous définissons une fonction discriminante $D\left(T_{\mathbf{c}}, T_{\mathbf{s}}\right)$. Celle-ci est la somme des pixels mal classés à $T_{\mathrm{p}}$ donné, où $T_{\mathrm{c}}$ et $T_{\mathrm{s}}$ sont respectivement les seuils sur les images calcium et silicium :

$$
D\left(T_{\mathrm{c}}, T_{\mathrm{s}}\right)=N_{\mathrm{o}}+N_{\mathrm{ps}}+N_{\mathrm{pc}}+N_{\mathrm{cs}}+N_{\mathrm{pcs}}
$$

avec $-N_{\mathrm{o}} \quad$ pixels qui ne sont ni porosité, ni calcium, ni silicium.

$-N_{\mathrm{pc}}$ pixels communs à la porosité et au calcium.

$-N_{\mathrm{ps}} \quad$ pixels communs à la porosité et au silicium.

$-N_{\mathrm{cs}} \quad$ pixels communs au calcium et au silicium.

$-N_{\text {pes }}$ pixels communs à la porosité, au calcium et au silicium.

La superposition est d'autant meilleure que $D\left(T_{\mathfrak{c}}, T_{\mathbf{s}}\right)$ est faible. La détection du minimum de $D\left(T_{\mathrm{c}}, T_{\mathrm{s}}\right)$ est un problème d'optimisation sans contrainte. La forme de $D\left(T_{\mathrm{c}}, T_{\mathrm{s}}\right)$ pour $T_{\mathrm{p}}=105$ (valeur donnée par la méthode du maximum d'entropie pour l'image étudiée) et pour $T_{\mathrm{c}}$ et $T_{\mathrm{s}}$ variant de 60 à 160 est représentée sur les figures $6 \mathrm{a}$ et $6 \mathrm{~b}$. A cette échelle, on ne perçoit qu'un seul minimum, mais à une échelle inférieure (tous les deux niveaux de gris) des minima locaux apparaissent. Pour rechercher le minimum, la méthode du simplex [10] a été utilisée, elle converge vers un minimum très proche du minimum absolu. 


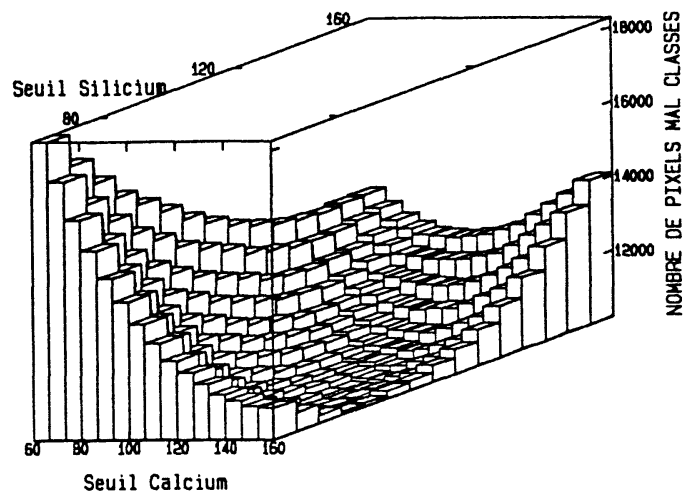

a)

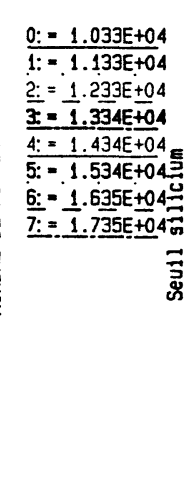

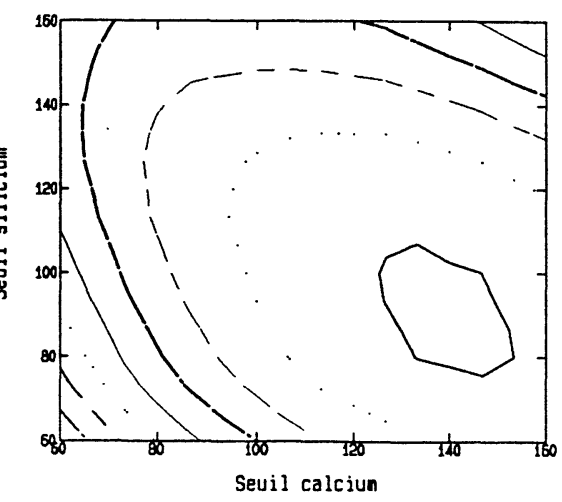

b)

Fig. 6. - Fonction discriminante suivant les variables $T_{\mathrm{c}}$ et $T_{\mathrm{s}}$. a) Représentation 3D. b) Représentation en courbes de niveaux.

[Discriminating function $v s . T_{\mathrm{c}}$ and $T_{\mathrm{s}}$. a) $3 \mathrm{D}$ plot. b) Level curves.]

\section{Résultats.}

Nous avons comparé les résultats obtenus par la minimisation de la fonction discriminante $D\left(T_{\mathbf{c}}, T_{\mathbf{s}}\right)$ à $T_{\mathbf{p}}$ donné à ceux fournis par d'autres méthodes :

- seuillage séparé sur les trois images par minimum d'erreur Bayésienne,

- seuillage séparé par maximum d'entropie,

- seuillage séparé par modification d'histogramme,

- fonction discriminante globale $D\left(T_{\mathrm{p}}, T_{\mathrm{c}}, T_{\mathrm{s}}\right)$ précédemment étudiée [11] où les trois seuils entrent en ligne de compte pour déterminer la minimum de pixels mal classés.

Les valeurs des seuils obtenus par ces méthodes sont comparées tableau II. Les figures 7-1a à $7-6 c$ représentent les trois images après seuillage par les divers seuils calculés. La figure 8 montre les différents pixels mal classés pour le vecteur seuil correspondant au minimum de $D\left(T_{\mathrm{c}}, T_{\mathrm{s}}\right)$. On peut voir qu'ils se situent principalement aux frontières.

Tableau II. - Comparaison des seuils.

[Comparison of the thresholds.]

\begin{tabular}{|c|r|r|r|}
\hline & $T_{\mathbf{p}}$ & $T_{\mathbf{c}}$ & $T_{\mathbf{s}}$ \\
\hline Min. erre. & 88 & 55 & 55 \\
\hline Max. entr. & 105 & 105 & 127 \\
\hline Mod. hist. & 99 & 128 & 103 \\
\hline$D\left(T_{\mathbf{p}}, T_{\mathbf{c}}, T_{\mathbf{s}}\right)$ & 145 & 133 & 71 \\
\hline$D\left(T_{\mathbf{c}}, T_{\mathbf{s}}\right)$ & 105 & 130 & 89 \\
\hline
\end{tabular}



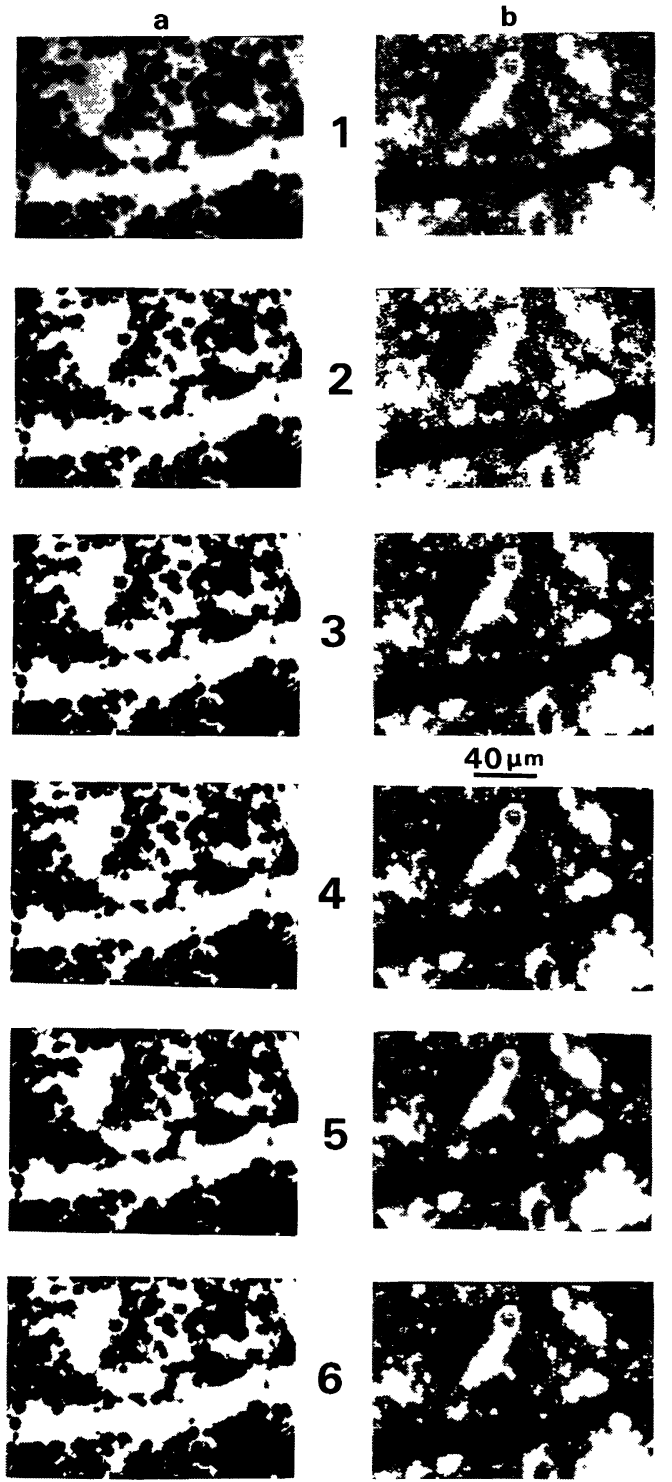
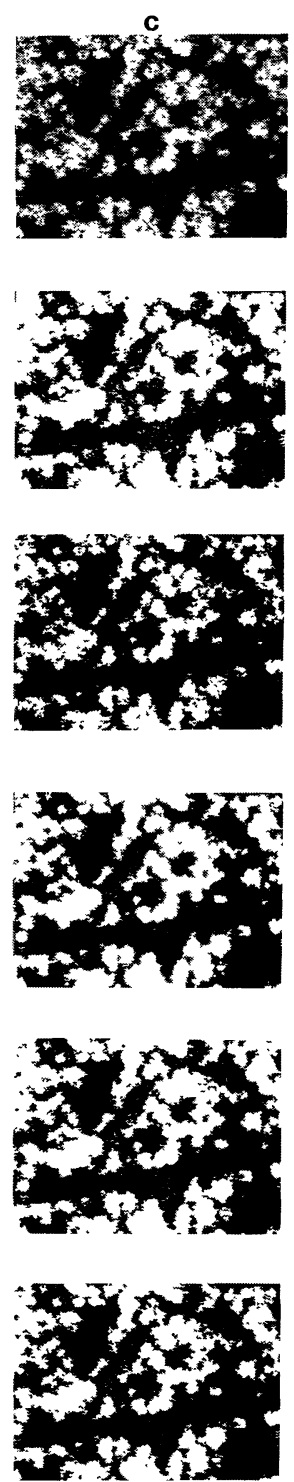

Fig. 7. - Extraction des divers composants suivant les méthodes de seuillage : a, b, c, désignent les trois composants considérés (porosité, calcium, silicium) 1) images originales, 2) méthode du minimum d'erreur bayésienne, 3 ) méthode du maximum d'entropie, 4) méthode de la modification d'histogramme, 5) fonction discriminante $\left.\left(T_{\mathrm{p}}, T_{\mathrm{c}}, T_{\mathrm{s}}\right), 6\right)$ fonction discriminante $\left(T_{\mathrm{c}}, T_{\mathrm{s}}\right)$.

[Pictures showing the different components extracted using various threshold methods: $a, b$ and $c$ ) the components (porosity, calcium and silicon). 1 to 6) original images and segmented images: 1) original images, 2) Bayesian minimum error method, 3) maximum of entropy method, 4) histogram modification method, 5) discriminating function $v s . T_{\mathrm{p}}, T_{\mathrm{c}}$ and $\left.T_{\mathrm{s}}, 6\right)$ discriminating function $v s . T_{\mathrm{c}}$ and $T_{\mathrm{s}}$.] 


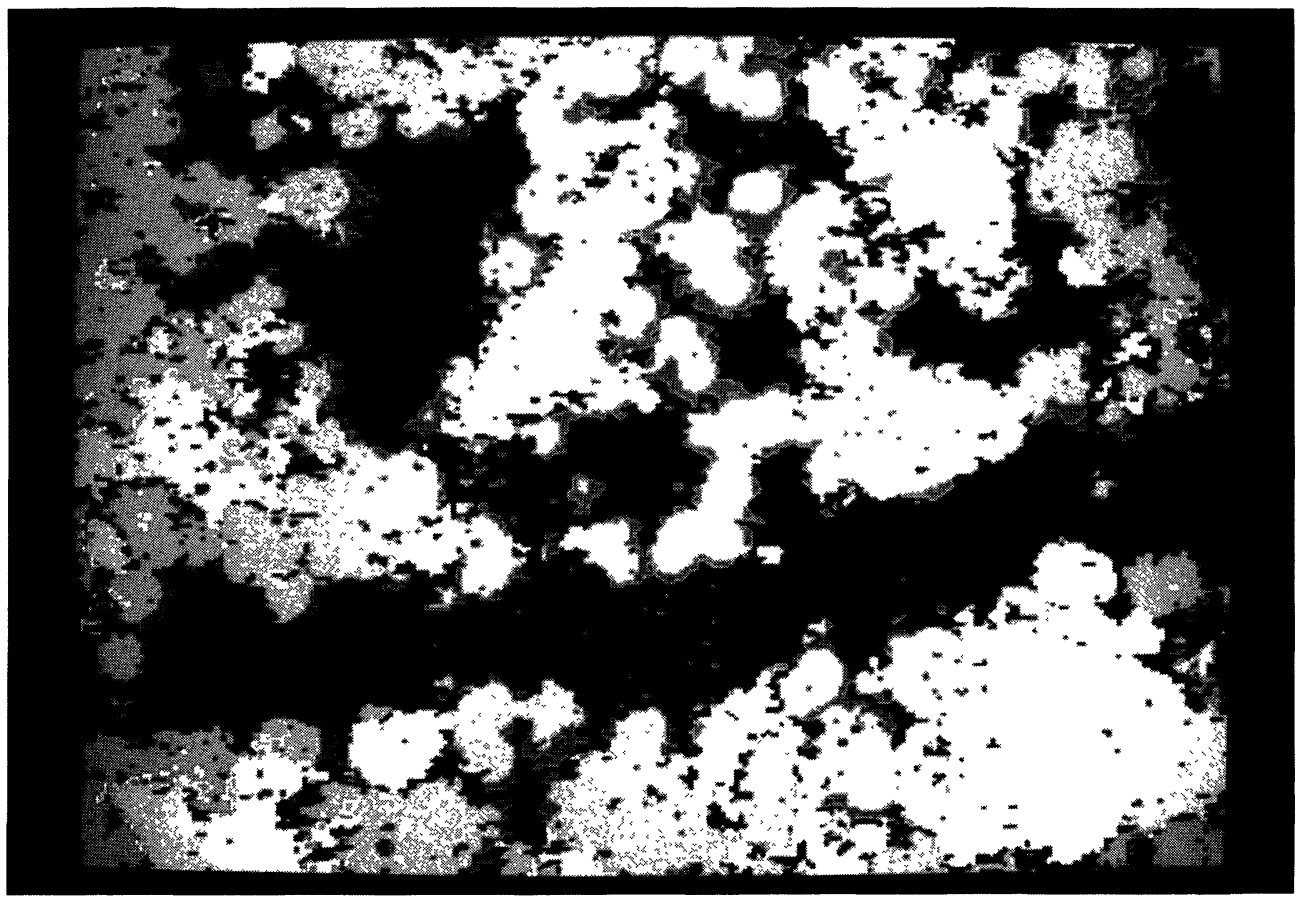

Fig. 8. - Les différentes classes de pixels. (La photo couleur est disponible chez les auteurs). [The different classes of pixels. The color photograph is available to the authors.]

Le tableau III donne les constituants trouvés par les différentes méthodes de seuillage. $\Sigma_{\mathrm{mc}}$ représente la somme des pixels mal classés. $N_{\mathrm{p}}, N_{\mathrm{c}}$ et $N_{\mathrm{s}}$ sont les pixels relatifs à chaque élément de la pierre c'est à dire la porosité, le calcium ou le silicium. On peut les qualifier de pixels bien classés.

Tableau III. - Répartition des classes de pixels suivant les différentes méthodes de seuillage. [Pixel classes $v s$. threshold methods.]

\begin{tabular}{|c|c|c|c|c|c|c|c|c|c|}
\hline & $N_{\mathrm{p}}$ & $N_{\mathrm{c}}$ & $N_{\mathbf{s}}$ & $N_{\mathbf{o}}$ & $N_{\mathrm{pc}}$ & $N_{\mathrm{ps}}$ & $N_{\mathbf{c s}}$ & $N_{\mathrm{pcs}}$ & $\Sigma_{\mathbf{m c}}$ \\
\hline Min. Erre. & 18047 & 12072 & 13975 & 509 & 2529 & 5740 & 11050 & 1614 & 21442 \\
\hline Max. entr. & 23482 & 14989 & 15210 & 7718 & 1047 & 1382 & 1673 & 35 & 11855 \\
\hline Mod. Hist. & 23176 & 12704 & 18930 & 5956 & 670 & 2748 & 1317 & 35 & 10726 \\
\hline$D\left(T_{\mathrm{p}}, T_{\mathbf{c}}, T_{\mathrm{s}}\right)$ & 19262 & 11853 & 24373 & 5345 & 157 & 2340 & 2194 & 12 & 10048 \\
\hline$D\left(T_{\mathbf{c}}, T_{\mathbf{s}}\right)$ & 22329 & 12255 & 20442 & 4791 & 590 & 3436 & 1649 & 44 & 10510 \\
\hline
\end{tabular}

Les techniques de seuillage étudiées donnent des résultats très différents. Des critères sont donc nécessaires pour estimer leurs qualités respectives. 
Un premier critère peut être jugement visuel. En observant les figures 7, on peut analyser les résultats sur les différentes images : élevée,

- pour l'image porosité, le minimum de $D\left(T_{\mathbf{p}}, T_{\mathbf{c}}, T_{\mathbf{s}}\right)$ donne une valeur pour $T_{\mathbf{p}}$ beaucoup trop

- pour l'image X calcium, le minimum d'erreur Bayésienne surévalue largement le calcium, le maximum d'entropie aussi dans une moindre mesure,

- pour l'image X silicium, hormis le minimum d'erreur Bayésienne, toutes les méthodes donnent des résultats corrects, le maximum d'entropie étant la meilleure.

De cette première analyse, seuls la fonction discriminante à $T_{\mathrm{p}}$ donné, le maximum d'entropie et la modification d'histogramme conviendraient pour les trois images.

Un autre critère moins subjectif peut être le nombre de pixels mal classés après seuillage. De ce point de vue, le minimum de $D\left(T_{\mathfrak{c}}, T_{\mathrm{s}}\right)$ donne de meilleurs résultats que les trois méthodes de seuillage direct car elle prend en compte la complémentarité des images en minimisant le nombre de pixels mal classés.

\section{Exploitation des résultats.}

L'exploitation des images seuillées permet de fournir à un expert des renseignements sur la pierre concernant sa porosité (pourcentage, caractéristiques géométriques...) et la granulométrie de ses constituants. Les seuils de binarisation des images sont ceux trouvés par la méthode du minimum de $D\left(T_{\mathbf{c}}, T_{\mathbf{s}}\right)$.

6.1 PourCENTAgE DES COMPOSANTS. - Le pourcentage des composants est donné tableau IV. Il conviendrait d'affecter les pixels mal classés à un des trois constituants en appliquant des critères locaux. Ce traitement postérieur n'est pas “implémenté”. La précision obtenue est suffisante pour les applications actuelles.

Tableau IV. - Les constituants de la pierre et les pixels mal classés.

[Stone components and poorly classified pixels.]

\begin{tabular}{|c|c|}
\hline porosité & $35 \%$ \\
\hline calcium & $18 \%$ \\
\hline silicium & $31 \%$ \\
\hline mal classés & $16 \%$ \\
\hline
\end{tabular}

On peut remarquer que cette pierre est très poreuse par nature (porosité volumique de 30 à $50 \%$ en moyenne). La circulation de l'eau, mécanisme physico-chimique principal de l'altération, dissout peu à peu le ciment de la pierre. Le pourcentage de calcium nous renseigne sur la solidité de matériau.

L'échantillon analysé, qui ne contient que $18 \%$ de calcium, provient d'une pierre altérée. En effet, pour une pierre de tuffeau saine la proportion de calcium est soit égale soit supérieure à celle de silicium. 
6.2 ANISOTROPIE DE LA POROSITÉ. - L'anisotropie des zones constituant la porosité peut être étudiée en traçant la rose des intercepts [12]. Ce tracé consiste à représenter en coordonnées polaires, le nombre de fois où l'on rencontre, en balayant dans une direction donnée l'image porosité binarisée, un couple de pixels qui correspond à une frontière porosité-particule. Plus ce nombre est élevé, plus la circulation d'un liquide sera difficile dans cette direction et inversement. Ce renseignement permet de déduire la direction du lit de la pierre, information fort utile lors d'un remplacement d'un élément de l'édifice.

La porosité de l'échantillon étudié est pratiquement isotrope, car la rose des intercepts pour l'image porosité (Fig. 9) est approximativement circulaire. A cette échelle d'analyse, il n'est donc pas possible de déduire une direction privilégiée pour l'écoulement.

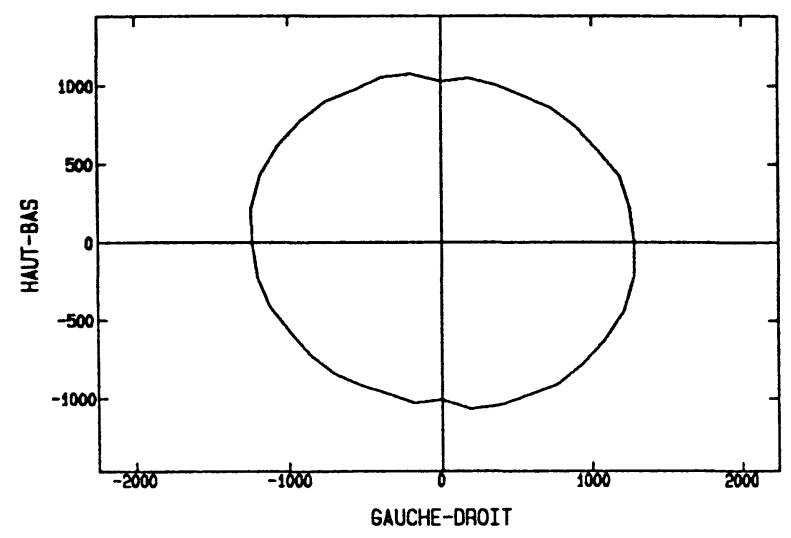

Fig. 9. - Rose des intercepts de l'image porosité.

[Intercept rose of the porosity image.]

6.3 GRANULOMÉTRIE DES CONSTITUANTS. - La nature granulométrique des particules peut être approchée en traçant le nombre de pixels blancs d'une image binaire après érosions successives [13]. La forme de la courbe représentant le nombre de pixels blancs restant, en fonction du nombre d'érosions, permet d'évaluer la taille moyenne des particules. Une décroissance rapide de la courbe correspond à des particules de petite taille et réciproquement. Ceci nous permet de savoir si l'altération est avancée ou non, plus les particules restantes sont de grandes tailles, plus l'édifice devient fragile. En effet, lorsque le ciment assurant la cohésion du matériau est dissout, la partie restante formée des cristallites les plus gros (quartz, mica, oxydes, etc...) est fragile, voire poudreuse.

Les courbes (Fig. 10) montrent que pour nos images, les particules de silicium sont en moyenne de tailles inférieures à celles de calcium car la décroissance est plus rapide pour le silicium. Ceci signifie que les petites particules de calcium sont déjà dissoutes et que le silicium est prépondérant dans le ciment de la pierre. L'échantillon de pierre étudié est donc en cours d'altération.

\section{Conclusion.}

Cet article décrit une méthode de traitement de trois images de la même scène qui consiste à utiliser l'information à priori concernant leur complémentarité. Elle a été comparée à d'autres 


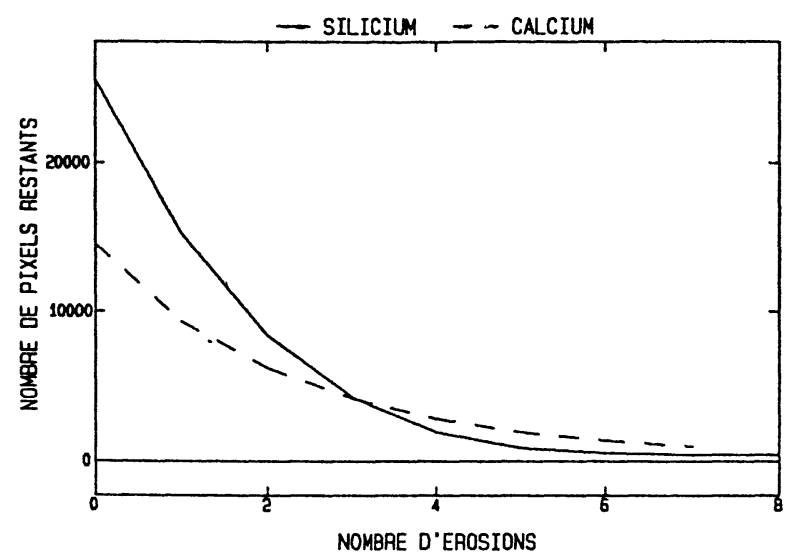

Fig. 10. - Evolution de la surface totale des particules contenant du calcium ou de la silice en fonction du nombre d'érosions binaires des images correspondantes.

[Size of the calcareous and silica area $v s$. number of binary erosions on the corresponding images.]

méthodes de seuillage et donne les meilleures résultats d'un point de vue de la superposition des trois images binaires.

Cette technique permet de séparer correctement les différents composants d'une pierre analysée par M.E.B. afin d'étudier ses caractéristiques. Les résultats permettent d'évaluer la porosité et la granulométrie des composants. Appliquées à des pierres de monuments historiques, elle fournit des données nécessaires à un expert pour apprécier l'état de l'édifice et pour remplacer certaines parties abîmées [14].

Les résultats pourraient être actuellement améliorés sur le plan de l'acquisition des images. En effet, il est à présent possible de ne pas passer par des films polaroïds pour améliorer la qualité des informations et résoudre les problèmes de calage.

De plus, pour la pierre de tuffeau, il existe de rares particules qui ne sont ni calcaires ni siliceuses (les oxydes de fer par exemple). Une des améliorations possibles serait de prendre en compte cette connaissance a priori lors de la séparation des particules en deux classes. Lors de la recherche du minimum de $D\left(T_{\mathrm{c}}, T_{\mathrm{s}}\right)$, il faudrait retrancher à $N_{\mathrm{o}}$ ces particules marginales, ce qui réduirait en conséquence le pourcentage de pixels mal classés.

\section{Bibliographie}

[1] EberhaRT J.P., Analyse structurale et chimique des matériaux (Ed. Dunod 1989).

[2] HEINRICH K. et al, Use of Monte Carlo calculations in electron probe microanalysis and scanning electron microscopy, U.S. National bureau of standards (1976).

[3] Maurice F., MENy L. et Tixier R., Microanalyse et Microscopie Electronique à balayage (Les éditions de physique, 1978).

[4] SAHOO P.K., SOLTANI S., WONG A.K.C. and CHEN Y.C., A survey of thresholding techniques Comput. vision, graphics and image processing 41 (1988) 233-260.

[5] KITTLER J. and IllingwORTH J., Minimum error thresholding Pattern Recognition 19 (1986) 41-47.

[6] PRATT W.K., Digital image processing, John Wiley and sons (1978).

[7] PUN T., Entropic thresholding, a new approach Comput. graphics and image processing 16 (1981) 210 239. 
[8] KAPUR J.N., SAHOO P.K. et WONG A.K.C., A new method for grey-level picture thresholding using the entropy of the histogram Comput. Vision, Graphics and image processing 29 (1985) 273-285.

[9] WESZKA J.S. and ROSENFELD A., Histogram modification for threshold selection IEEE Trans. Systems, Man, Cybernetics SMC-9 (1979) 38-51.

[10] DANIELS R.W., An introduction to numerical methods and optimization techniques (North-Holland Ed. 1978).

[11] HARBa R., JACQUET G. and RAUTUREAU M., Study of stones by image processing, Barcelone EUSIPCO 90 (1990) 999-1002.

[12] COSTER M. et ChERMANT J.L., Précis d'analyse d'images (Ed. du CNRS 1985).

[13] JEULIN D., Mathematical morphology and material image analysis (Ed. Scanning Microscopy International) 2 (1988) pp. 165-184.

[14] RAUTUREAU M., Connaissance du tuffeau, microscopie électronique et analyse d'images, quelques concepts physiques d'étude, in Advanced Workshop in analytical methodologies for the investigation of damaged stones (Pavie, Septembre 1990). 